\title{
Alkali P-Nitrophenolates for Short Wavelength Laser Generation
}

\author{
M.Thangaraj \\ PSNA College of Engineering, \\ Dindigul \\ Tamil Nadu, India
}

\author{
G. Ravi \\ Alagappa University \\ Karaikudi \\ Tamil Nadu, India
}

\author{
T. Mahalingam \\ Dept. of Physics, \\ Karunya University \\ Coimbatore, India
}

\begin{abstract}
Single crystals of alkali p-Nitrophenolates namely sodium p-nitrophenolate dihydrate (SPNP), potassium p- nitrophenolate monohydrate (PPNP) and lithium p-nitrophenolate trihydrate (LPNP) using Group I elements (Na, K, Li) and p-nitrophenols were grown by solvent evaporation method. Single crystal XRD analysis shows that SPNP and LPNP crystallize in noncentrosymmetric space group while PPNP is centrosymmetric. Using Autox software, all the peaks in the recorded powder XRD spectrum of the samples were identified and indexed. The FT - IR spectra of the sample reveals the characteristic vibrations of the functional groups present in alkalinitrophenolates. A weak absorption band around the region $1589-1641 \mathrm{~cm}^{-1}$ confirms the presence of the phenolic ring. A broad intermolecular hydrogen bonded $\mathrm{OH}$ stretching at $3325 \mathrm{~cm}^{-1}$ of $\mathrm{p}$ - nitrophenol is shifted which shows the increase in the polarizable nature of p-nitrophenol and thus easily forms a metal (sodium/ potassium/ lithium) coordination compound. UV-Vis spectrum shows that all the crystals are transparent above $400 \mathrm{~nm}$ and has a wide optical window in the visible region. Intense absorption peak in the UV region may be due to the colored nature of the compound. Addition of metal ion (sodium/potassium) modifies the optical transparency of the original molecule (p-nitro phenol) and consequently introduces a bath chromic shift of 90/40 nm in the crystal transparency of the samples. Kurtz powder technique result shows that the relative SHG efficiency of SPNP and LPNP was nearly 5 and 9.25 times of KDP.
\end{abstract}

Key Words: p-Nitrophenol, SHG Efficiency, Alkali Metals.

\section{INTRODUCTION}

The p-nitrophenolate derivatives are the other forms of metalorganic coordination complexes. The prototype organic NLO material contains one or more delocalized bonds, typically a ring structure like benzene. When substituted with donor and acceptor at the para position, the bonds get aligned and thus have a large induced dipole moment under the influence of electromagnetic fields. p-nitrophenol is a typical example for the above strategy [1]. It has often been found that such structures, when packed as crystals tend to be centrosymmetric, thus leading to macroscopically vanishing dipole moment. However, when it reacts with the bases, the resulting compound is acidic with a non-vanishing dipole moment for the donor-acceptor $\pi$ conjugate systems [2,3]. The life time of an optical device (electro- optic modulators, parametric oscillator, etc.) is strongly dependent on mechanical and thermal properties of the nonlinear material. Organic materials are more fragile than inorganics owing to van der Waals bonds which maintain the cohesion of molecular packing. Based on these favorable properties, three nitrophenolates namely (i) sodium p-nitrophenolate dihydrate (SPNP), (ii) potassium p- nitrophenolate monohydrate (PPNP) and (iii) lithium p-nitrophenolate trihydrate (LPNP) have been synthesized and their optical properties were thoroughly investigated and reported.

\section{EXPERIMENTAL PROCEDURE}

\subsection{Synthesis and Crystal Growth}

The basic chemical reaction considered for synthesis is that a caustic alkali base (alkali hydroxide) will lose a proton in a weak acidic (p- nitrophenol) leading to the formation of the salt. Thus in the present investigation, the salt is obtained by dissolving p-nitrophenol in alkali hydroxide $(\mathrm{NaOH}, \mathrm{KOH}$, $\mathrm{LiOH})$ solution at $32^{\circ} \mathrm{C}$ in the molar ratio $1: 1: 30$ for p-nitrophenol, alkali hydroxide and double distilled water respectively. By cooling the solution to room temperature a yellow crystalline powder is obtained.

From solubility studies, methanol was found to be the best solvent to grow SPNP and PPNP, while water was chosen for LPNP. Then the prepared solutions were allowed to slow evaporation, after a period of seven days yellow coloured single crystals of SPNP, PPNP and LPNP were obtained.

\section{RESULTS AND DISCUSSION}

\subsection{Structural Analysis}

The grown crystals were subjected to single crystal X-ray diffraction using a Enraf Nonius CAD-4 single crystal X-ray diffractometer with $\mathrm{Cu} \mathrm{K} \alpha$ radiation $(\lambda=1.54060 \AA)$ to determine the unit cell dimensions. XRD analysis shows that SPNP crystal belongs to the orthorhombic system with a

noncentrsymmetric space group Ima2. The PPNP belongs to monoclinic system with a centrosymmetic space group $\mathrm{P} 2{ }_{1} / \mathrm{c}$, which does not exhibit second order NLO activity. Also LPNP crystallizes in monoclinic system with a noncentrsymmetric space group $\mathrm{Pa}$. Their cell dimensions are given in the Table 1 . All the unit cells have $(Z=4)$ tetra molecules and have a suppressed growth along $\mathrm{c}$ axis. 
It is interesting to note that elements in the complexes that are more electronegative induce more delocalization of electrons, favoring the noncentrosymmetric structure [4].

This is the reason why, PPNP is centrosymmetric while LPNP and SPNP are noncentrosymmetric as the electro negativity of alkali metals $\mathrm{Li}(0.98), \mathrm{Na}(0.93)$ and $\mathrm{K}(0.82)$ falls down as one move down the Group.

Table 1. Cell Parameters of Nitrophenolates

\begin{tabular}{|c|c|c|c|c|c|}
\hline $\begin{array}{c}\text { Sampl } \\
\mathbf{e}\end{array}$ & $\mathbf{a}(\AA)$ & b $(\AA)$ & c $(\AA)$ & $\begin{array}{c}\beta \\
(\mathbf{d e g})\end{array}$ & $\mathbf{V}\left(\AA^{3}\right)$ \\
\hline SPNP & $6.896(7)$ & $\begin{array}{l}19.709 \\
(6)\end{array}$ & $\begin{array}{c}6.443 \\
(3)\end{array}$ & 90 & $\begin{array}{l}875.68 \\
(9)\end{array}$ \\
\hline PPNP & $\begin{array}{c}10.561 \\
(3)\end{array}$ & $\begin{array}{c}7.379 \\
(2)\end{array}$ & $\begin{array}{c}11.299 \\
(4)\end{array}$ & $\begin{array}{c}117.8 \\
0\end{array}$ & $\begin{array}{c}778.72 \\
(3)\end{array}$ \\
\hline LPNP & $\begin{array}{c}10.838 \\
(8)\end{array}$ & $\begin{array}{c}7.519 \\
(4)\end{array}$ & $\begin{array}{c}11.31 \\
(4)\end{array}$ & 90.54 & $925.7(9)$ \\
\hline
\end{tabular}

The powder samples were subjected to powder XRD (Figure 1) analysis using a X'Pert diffractometer with $\mathrm{CuK} \alpha$ radiation $(\lambda=1.5418 \AA)$. The computer program AUTOX was used to index all the observed reflection in the XRD pattern and to calculate the corresponding lattice parameters. The lattice parameter evaluated from PXRD agrees very well with single crystal XRD data.

The figure 1C explains the comparision of particle size, lattice constant variation with the calcination temperature. In is observed from the figure that, particle size increases with increasing calcination temperatures and lattice constant decreases with temperatures.

\subsection{Molecular Characterization}

The FT - IR spectra of SPNP, PPNP and LPNP reveals that the characteristic vibrations of the functional groups present in nitrophenolates.

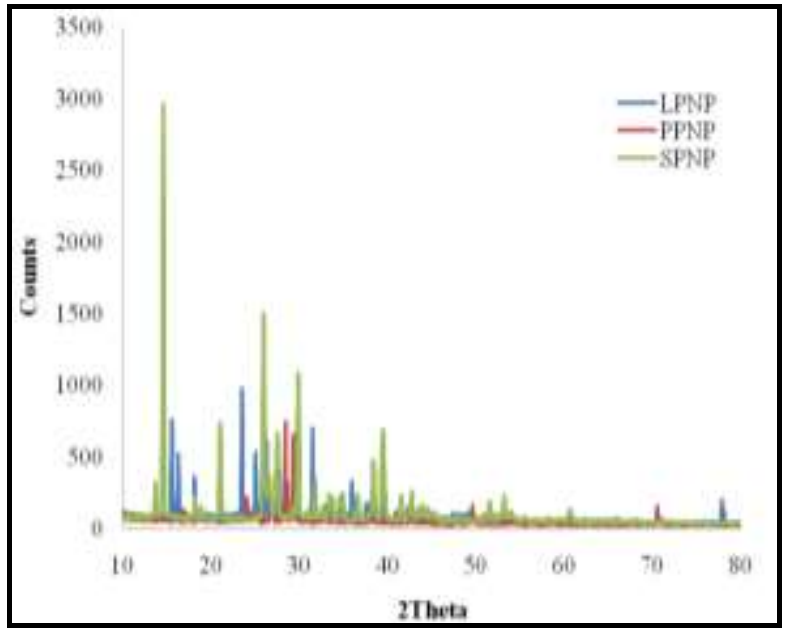

Figure 1 Powder XRD Pattern of Nitrophenolates.

The absorptions in the lower frequency region $\left(400-500 \mathrm{~cm}^{-1}\right)$ are due to the overtones of the fundamental vibrations of p-nitrophenolates. The vibrational frequencies of the functional groups present in alkali p-nitrophenolates are compared with the p- nitrophenol (PNP) in Table 2

Table 2. FT-IR bands of alkali p-nitrophenolates.

\begin{tabular}{|c|c|c|c|c|}
\hline \multicolumn{4}{|c|}{ Wavenumber $\left(\mathrm{cm}^{-1}\right)$} & \multirow{2}{*}{ Assignment } \\
\cline { 1 - 4 } PNP & LPNP & SPNP & PPNP & \\
\hline 3325 & 3408 & 3189 & 3211 & $v_{\mathrm{s}}(\mathrm{OH})$ \\
\hline 3060 & 3059 & - & $3045 *$ & $v_{\mathrm{s}}(\mathrm{C}-\mathrm{H})$ \\
\hline 1613 & 1666 & 1682 & 1665 & $\delta(\mathrm{O}-\mathrm{H})$ \\
\hline 1590 & 1573 & 1576 & 1601 & $v_{\mathrm{s}}\left(\mathrm{NO}_{2}\right)$ \\
\hline 1494 & 1495 & 1484 & 1467 & $v_{\mathrm{s}}(\mathrm{C}=\mathrm{C})$ \\
\hline 1326 & 1320 & 1302 & 1343 & $v_{\mathrm{as}}\left(\mathrm{NO}_{2}\right)$ \\
\hline 865 & 855 & 853 & 846 & $v_{\mathrm{as}}(\mathrm{C}=\mathrm{C})$ \\
\hline 817 & 822 & 814 & 757 & $\delta(\mathrm{C}-\mathrm{H})$ \\
\hline 710 & 705 & 703 & 699 & $\delta(\mathrm{C}=\mathrm{C})$ \\
\hline 642 & 647 & 644 & $629 *$ & $\delta(\mathrm{O}-\mathrm{H})$ \\
\hline- & 497 & 494 & 483 & $(\mathrm{Li} / \mathrm{Na} / \mathrm{K})$ \\
\hline
\end{tabular}

$$
\begin{aligned}
& v_{\mathrm{s}-} \text { symmetric stretching } \quad v_{\mathrm{as}}-\text { asymmetric stretching } \\
& \delta \text { - bending }
\end{aligned}
$$

A weak absorption band around the region 1589 $1641 \mathrm{~cm}^{-1}$ confirms the presence of the phenolic ring. The shift in broad intermolecular hydrogen bonded $\mathrm{OH}$ stretching at 3325 $\mathrm{cm}^{-1}$ increase the polarizable nature of p-nitrophenol to a higher order and it easily forms a metal coordination compound and hence confirms the molecular structure of the synthesized compounds. 


\subsection{Linear Optical Characterization}

Optical transmittance of the crystalline samples was recorded using a Varian Cary 5E UV-Vis-NIR spectrophotometer in the UV - Vis region. In SPNP single crystals, $60 \%$ optical transmittance occurs at $520 \mathrm{~nm}$ and it has a good optical transmittance window in the region 520-1500 nm. In PPNP, the intramolecular charge transfer between the donor and acceptor groups give an intense absorption band ( $\mathrm{EtOH}$ as solvent ) in the UV region with $\lambda_{\max }$ at $388 \mathrm{~nm}$ and $310 \mathrm{~nm}$. This may be due to the colored compound absorbing in the visible region. In LPNP, $80 \%$ transparency occurs at $450 \mathrm{~nm}$ and is almost transparent till $1500 \mathrm{~nm}$ (Figure 2). Addition of metal ion (sodium/potassium/lithium) modifies the optical transparency of the original molecule (p-nitro phenol) and consequently introduces a bath chromic shift of $90 / 40 \mathrm{~nm}$ in the crystal transparency.

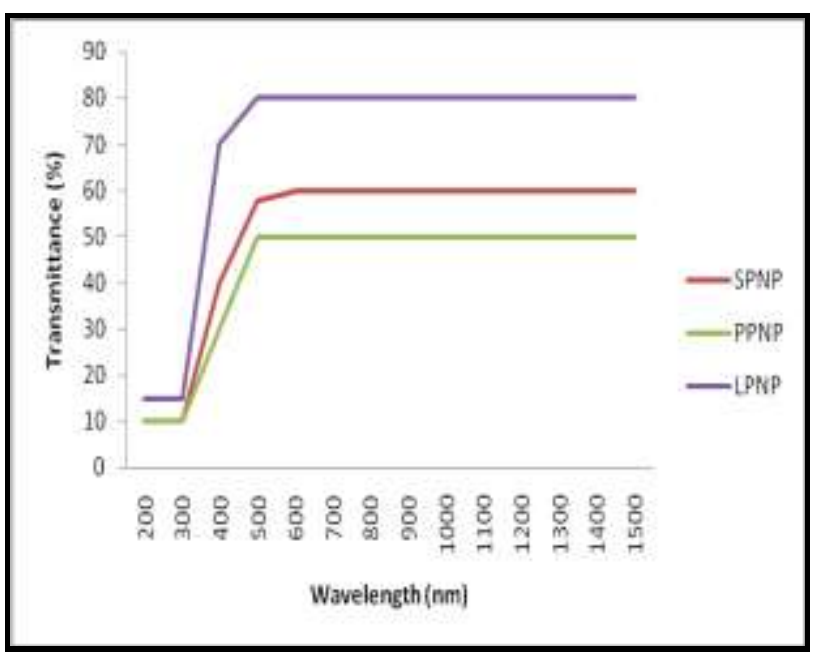

Figure. 2 Optical Transmittance of Nitrophenolates

\subsection{Non-linear Optical Characterization}

The synthesized salts of p-nitrophenolates were imposed for SHG test by Kurtz powder technique using a Q-switched Nd:YAG laser $(1064 \mathrm{~nm}, 10 \mathrm{~ns}, 10 \mathrm{~Hz})$. The test reveals that SPNP, LPNP exhibits SHG and no SHG signal was observed for PPNP. In SPNP and LPNP, metal atom is ionically bonded with p-nitrophenol introducing the noncentrosymmetry, which is an essential criterion for SHG. The results are compared with the pulverized KDP and the relative powder SHG efficiency was found to be 5 and 9.25 times greater than KDP for SPNP and LPNP respectively.

It is worthwhile to note that a material with smaller ionic radii has larger polarizability and hence larger nonlinear polarization[4]. Thus LPNP with lowest ionic radii alkali $(\mathrm{Li}=$ $0.76 \AA$ A) metal has higher SHG efficiency than other alkali

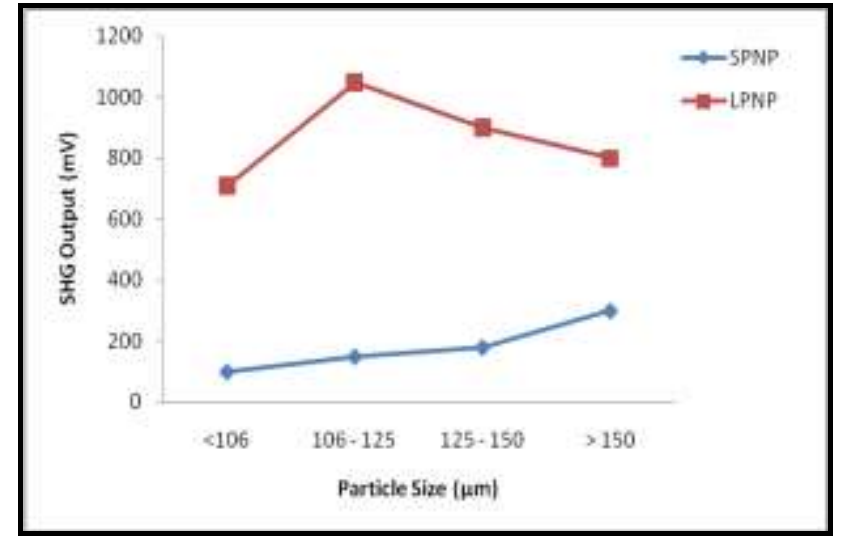

Figure. 3 Particle size vs. SHG output.

nitrophenolates. The intensity of the SHG output as a function of particle size was measured. The SHG output of SPNP increases with respect to the range of particle sizes, indicating the phase matchable character of the SPNP. While for LPNP, SHG output decreases with increase in particle size, showing the non-phase matchable nature of LPNP (Figure 3).

\section{CONCLUSION}

In the present investigation, attempts were made to synthesize certain semiorganic NLO materials based on nitrophenolates by acid-base reaction using alkali metals. Single crystals of considerable sizes were grown following the low temperature solution growth technique. X-ray diffraction studies reveal that SPNP and LPNP crystallize in the noncentrosymmetric space group with prominent crystallographic planes suitable for further studies. All the crystals exhibit a wide optical transmittance window $(450-1500 \mathrm{~nm})$ in the UV-Vis region irrespective of the substitutions. From the powder SHG efficiency studies the phase matchable SPNP and non phase matchable LPNP with high SHG efficiency (nearly 5 and 9.25 times of KDP) are found to be the potential candidates for the generation of short wavelength lasers by frequency doubling phenomena.

\section{REFERENCES}

[1] Chemla, D.S. Zyss, J. 1987, Nonlinear Optical Properties of Organic Molecules and Crystals. Vol $1 \& 2$.

[2] Brahadeeswaran, S. Venkataraman, V. Sherwood, N.J. Bhat, L.H. 1998, J. Mater. Chem. 8, 613.

[3] Milton Boaz, B. Leyo Rajesh, A. Xavier Jesu Raja, S SJerome Das, S.2004, J. Crystal Growth, 262, 531.

[4] Sabari Girisun, C. T. and Dhanuskodi, S.2010, Mater. Res. Bull. 45, 88. 